\title{
Frontal Sinus Schwannoma: Case Report and Literature Review
}

\author{
O. Benhoummad, Y. Labani, F. E. Rizkou, Y. Rochdi, and A. Raji
}

\section{ABSTRACT}

\begin{abstract}
Whilst schwannomas are mostly developed in the head neck area, the sinonasal involvement is extremely rare, especially those of the frontal sinus. We report a patient with sinonasal schwannoma, invading both frontal sinuses, anterior cranial fossa, the left anterior ethmoidal sinus, and the left nasal cavity. Radiologic imaging showed an isodensed, T2 isointense, T1 hypointense, with heterogeneous enhanced postcontrast mass with bone destruction. The patient underwent resection of the tumor via two approaches, external one and endonasal one. The histological diagnosis was consistent with a schwannoma. The follow-up was marked by the presence of diplopia without any signs of meningitis or cerebrospinal fluid rhinorrhoea.
\end{abstract}

Keywords: Schwannoma, frontal sinus, paranasal sinuses.

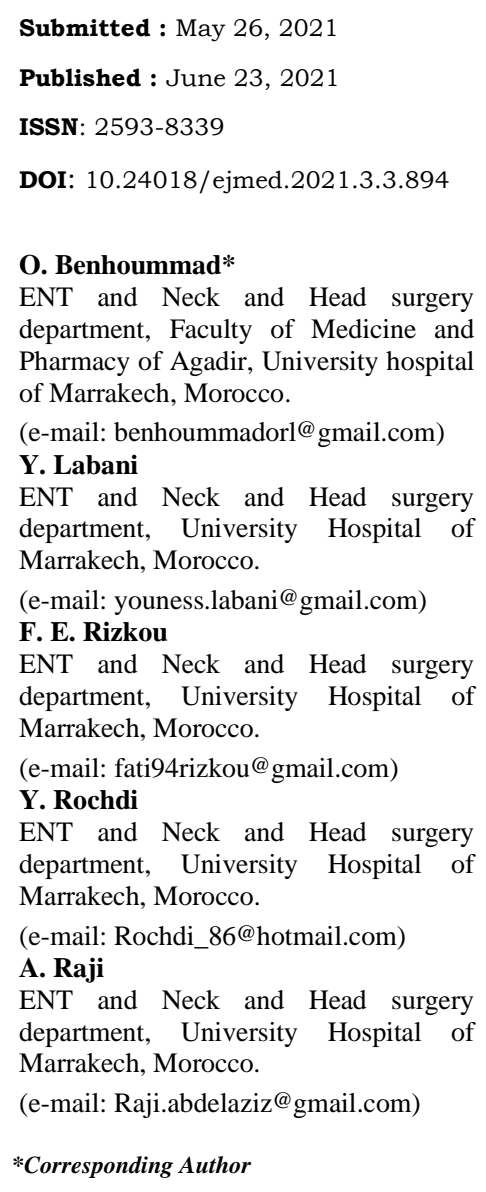

\section{INTRODUCTION}

Schwannoma is benign tumor arising from Schwann cells. The sinonasal location and in particularly the frontal one is rare. We report a new case of sinonasal schwannoma in a 33 year old male patient invading both frontal sinuses, anterior cranial fossa, the left anterior ethmoidal sinus, and the left nasal cavity.

\section{CASE REPORT}

A 33 year old patient, without no prior pathological history, presented for 18 months a gradually enlarging frontal swelling associated for the last 9 of those 18 months with headache and left nasal obstruction, without any sign fever or general state alteration. Endoscopic examination revealed a gray pinkish, smooth, firm, and soft tissue mass occupying the entire left nasal cavity arriving at the edge of the inferior turbinate and bulging into the nasopharynx Fig. 1, the contralateral nasal cavity appeared normal. The rest of the physical examination shows a frontal, firm, slightly painful, non-mobile, and well-defined swelling without any endobuccal expression or cervical adenopathy. The ophthalmologic and neurologic examinations were unremarkable.

CT scan and MRI showed an isodensed, T2 isointense, T1 hypointense, with heterogeneous enhanced postcontrast mass of both frontal sinuses, the left anterior ethmoidal sinus, and the left nasal cavity, extended anteriorly to the frontal soft tissue and posteriorly to the endocranial Fig. 2. This process caused bone destruction of the frontal sinus's anterior and posterior wall, anterior cranial fossa, and the interior wall of the left orbit.

The histologic and immunohistochemical studies of an endoscopic biopsy revealed a tumoral proliferation of spindle 
cell with regular elongated nuclei, without any nuclear atypia with intense PS-100 expression abling the schwannoma diagnosis Fig. 3.

The chirurgical intervention combined two approaches, external one; trans facial; through a mid-frontal incision, and endonasal one. The complete resection of the mass, that extends to the endocranial extradural level and left orbit, was performed with an endonasal frontal sinus drainage defining a Draf III. Reconstruction of the skull base was carried out by septal cartilage, abdominal fat, biological glue, and Surgicel Fig. 4. The anatomopathological study of the mass did indeed confirmed the diagnosis.

Postoperatively, the patient did present a diplopia without any signs of meningitis or cerebrospinal fluid rhinorrhoea.

\section{DISCUSSION}

Schwannoma is a rare benign tumor, emerging from the Schwann cells that forms the myelin sheath of peripherals nerves and it can be developed at any areas of the human body [1]. 25 to $40 \%$ of schwannomas take place in the head neck area [2] and less than $4 \%$ of those represents the sinonasal location [3]. The pathogenesis of schwannomas remains uncertain, but it can affiliated to the sensory branches of the trigeminal nerve or the perivascular nerve plexuses [4].

Frontal sinus schwannoma is uncommon; an article published by Lyer and Al in 2018 has reported 2 cases of frontal sinus schwannoma and reviewed 10 others in literature [5]. A review of 94 cases of sinonasal and anterior skull base schwannomas allowed to specify the different characteristics of this affection [6]. This disease affects subjects from age range of 14 to 81 years with an average age of 40 years old. The location was exclusively sinonasal in $46 \%$ of the cases. The main reported signs are nasal obstruction, headache, olfactory disorder such as hyposmia and anosmia. On CT scan the typical appearance of these tumors is marked by calcifications or bone destruction, while on MRI they were often appreared in iso or hypo signal T1 and hypersignal $\mathrm{T} 2$ with heterogeneous enhancement after the injection of gadolinium. That been said, the clinical/radiological aspect of this disease is not specific and may mimic different differential diagnoses such as esthesioneuroblastoma or olfactory meningioma. The anatomopathological and immunohistochemical studies allow the diagnoses; revealing a proliferation of spindleshaped cells, with small nuclei gathered in a cell population either in order called Antoni A or sometimes in disorder called Antoni B, with a positive immunostaining for the $\mathrm{S}$ 100 protein [7].

The treatment is based on the complete surgical resection, the partial one is performed when the tumor adheres to noble neurovascular structures. The external bicoronal approach was once the most used procedure for the resection of tumors invading the anterior level of the skull base. Nowadays, the use of the endoscopic approach combined with less invasive external route is more frequent [8].

The particularity of our case is the resort to the endoscopic approach combined with an external route mid frontal. This choice was made due to the tumor's extension to the frontal soft tissues, the two frontal and ethmoidal sinuses, the anterior cranial fossa and the left nasal cavity.

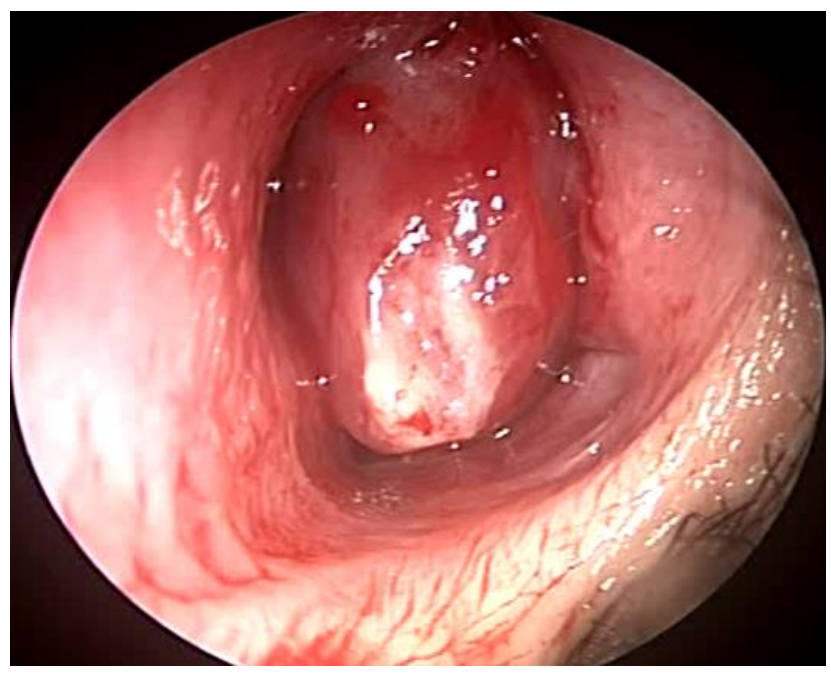

Fig. 1. Endoscopic view of the tumor.

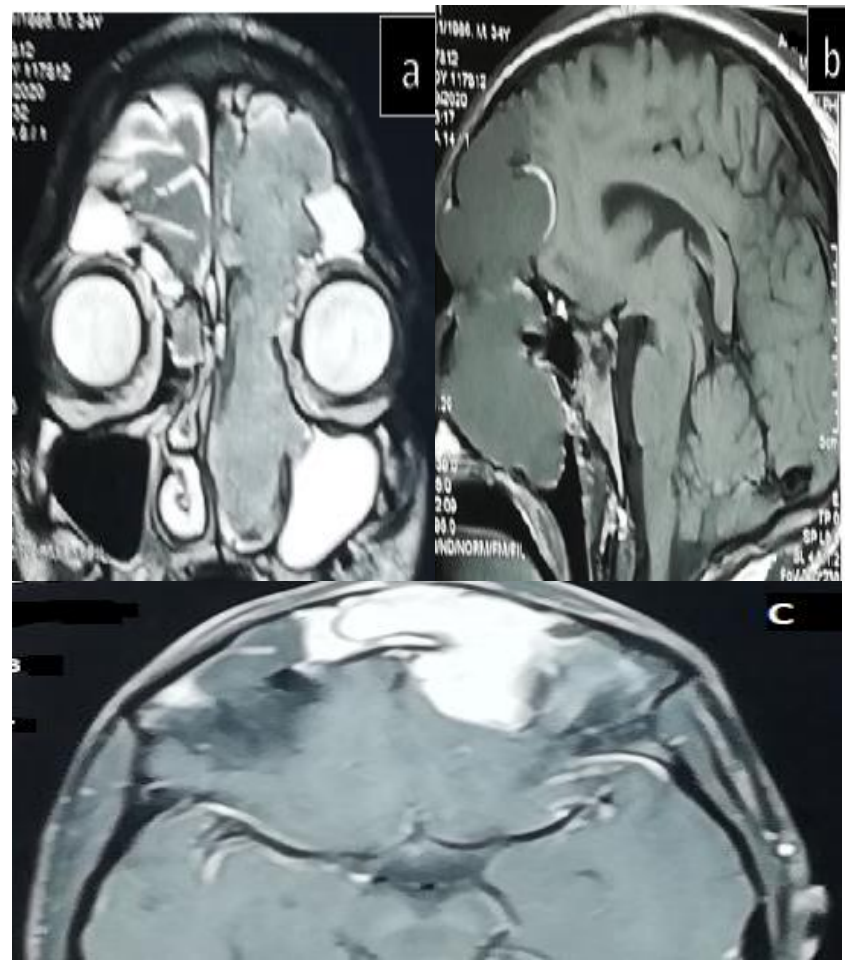

Fig. 2. Coronal T2 MRI showing orbital and endocranium extension of the tumor (a), sagittal T1 MRI showcasing endocranium extension (b), axial gadolinium- enhanced T1 weighted MRI showing endocranium extension (c).

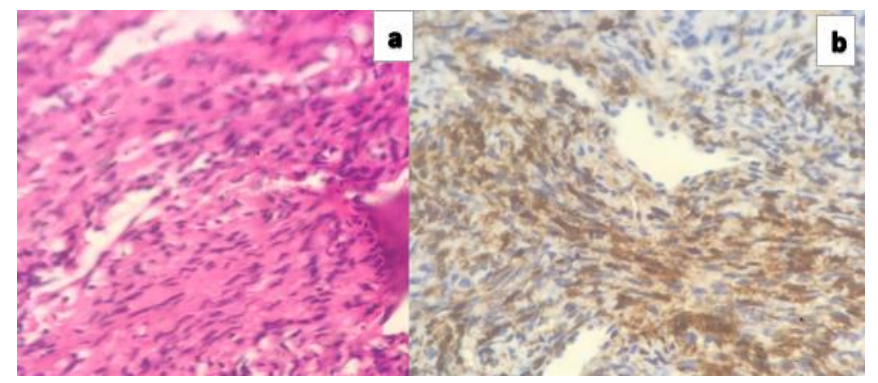

Fig. 3. Microscopic appearance of tumor (a), positive immunohistochemical staining for PS-100 (b). 


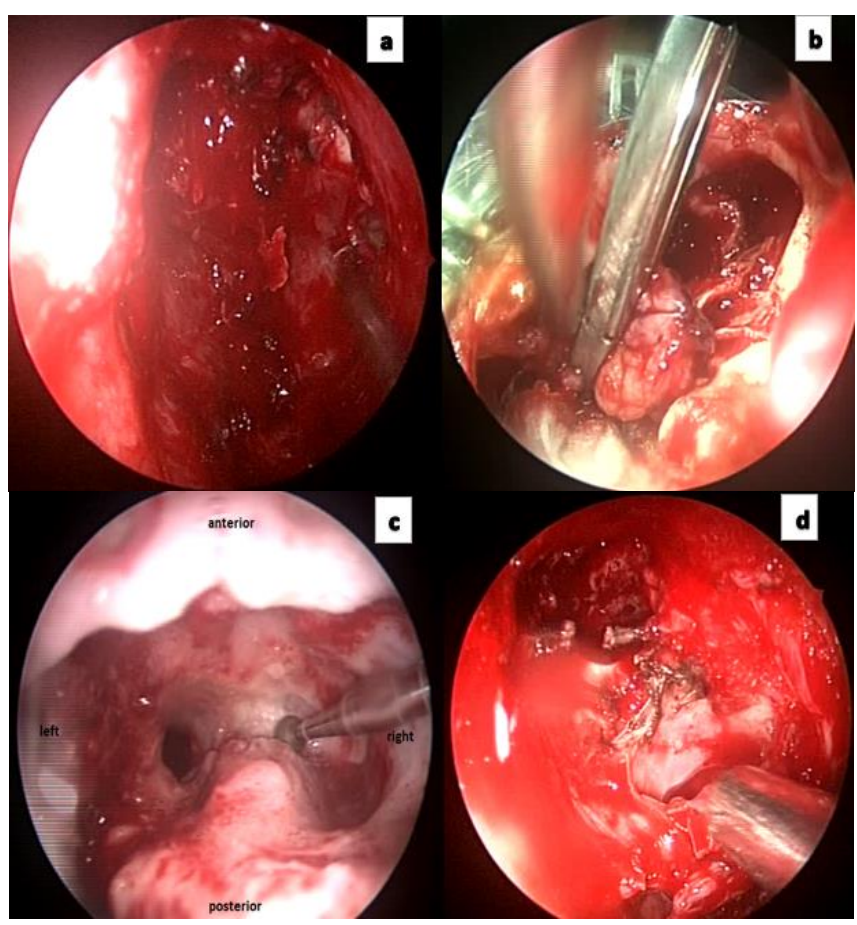

Fig. 4. perioperative view of endoscopic resection of tumor(a), perioperative view of tumor resection by external approach(b), frontal endoscopic view of Draf III procedure(c), perioperative view of the reconstruction of skull base (d).

\section{CONCLUSION}

The standard treatment of sinonasal schwannomas reside in the complete surgical resection. The development of the endoscopic surgery permitted the employment of miniinvasive routes which leads to the achievement of less complications.

\section{REFERENCES}

[1] N. Subramanian, N. Sood, and W. Ramalingam, "Large Sinonasal Schwannoma with Skull Base Defect: A Case Report," Indian J Otolaryngol Head Neck Surg, 2019. 71(Suppl 1): p. 887-889.

[2] Zhou, H., et al., "Schwannoma of the Sinonasal Tract and the Pterygopalatine Fossa with or without Intracranial Extension," ORL J Otorhinolaryngol Relat Spec, 2015. 77(2): p. 61-9.

[3] Mangubat, E.Z., et al., "Frontal sinus schwannoma: case report and review of literature," Skull Base Rep, 2011. 1(1): p. 17-22.

[4] Kumar, S. and C. Sayoo, "Sinonasal Schwannoma: A Rare Sinonasal Neoplasm,” Indian J Otolaryngol Head Neck Surg, 2017. 69(3): p. 425 427

[5] Iyer, A., et al., "Schwannomas of the Frontal Sinus: Cases and Review of the Literature," World Neurosurg, 2018. 110: p. 485-491.

[6] Sunaryo, P.L., et al., "Schwannomas of the sinonasal tract and anterior skull base: a systematic review of 94 cases," Am J Rhinol Allergy, 2014. 28(1): p. 39-49.

[7] Gencarelli, J., et al., "Atypical presentation of sinonasal cellular schwannoma: a nonsolitary mass with osseous, orbital, and intracranial invasion," J Neurol Surg Rep, 2014. 75(1): p. e144-8.

[8] Blake, D.M., et al., "Endoscopic endonasal resection of sinonasal and anterior skull base schwannomas," J Clin Neurosci, 2014. 21(8): p 1419-23. 